\title{
New "light" for one-world approach toward safe and effective control of animal diseases and insect vectors from leishmaniac perspectives
}

\author{
Kwang Poo Chang * (D), Bala K. Kolli and the New Light Group
}

\begin{abstract}
Light is known to excite photosensitizers (PS) to produce cytotoxic reactive oxygen species (ROS) in the presence of oxygen. This modality is attractive for designing control measures against animal diseases and pests. Many PS have a proven safety record. Also, the ROS cytotoxicity selects no resistant mutants, unlike other drugs and pesticides. Photodynamic therapy (PDT) refers to the use of PS as light activable tumoricides, microbicides and pesticides in medicine and agriculture.

Here we describe "photodynamic vaccination" (PDV) that uses PDT-inactivation of parasites, i.e. Leishmania as whole-cell vaccines against leishmaniasis, and as a universal carrier to deliver transgenic add-on vaccines against other infectious and malignant diseases. The efficacy of Leishmania for vaccine delivery makes use of their inherent attributes to parasitize antigen (vaccine)-presenting cells. Inactivation of Leishmania by PDT provides safety for their use. This is accomplished in two different ways: (i) chemical engineering of PS to enhance their uptake, e.g. Si-phthalocyanines; and (ii) transgenic approach to render Leishmania inducible for porphyrinogenesis. Three different schemes of Leishmania-based PDV are presented diagrammatically to depict the cellular events resulting in cell-mediated immunity, as seen experimentally against leishmaniasis and Leishmania-delivered antigen in vitro and in vivo. Safety versus efficacy evaluations are under way for PDT-inactivated Leishmania, including those further processed to facilitate their storage and transport. Leishmania transfected to express cancer and viral vaccine candidates are being prepared accordingly for experimental trials.

We have begun to examine PS-mediated photodynamic insecticides (PDI). Mosquito cells take up rose bengal/cyanosine, rendering them light-sensitive to undergo disintegration in vitro, thereby providing a cellular basis for the larvicidal activity seen by the same treatments. Ineffectiveness of phthalocyanines and porphyrins for PDI underscores its requirement for different PS. Differential uptake of PS by insect versus other cells to account for this difference is under study.

The ongoing work is patterned after the one-world approach by enlisting the participation of experts in medicinal chemistry, cell/molecular biology, immunology, parasitology, entomology, cancer research, tropical medicine and veterinary medicine. The availability of multidisciplinary expertise is indispensable for implementation of the necessary studies to move the project toward product development.
\end{abstract}

Keywords: Photosensitizers, Leishmania, Mosquito, Photodynamic therapy, Photodynamic vaccination, Photodynamic insecticide

\footnotetext{
* Correspondence: kwangpoo.chang@rosalindfranklin.edu

Department of Microbiology/Immunology, Chicago Medical School/Rosalind

Franklin University of Medicine and Science, 3333 Green Bay Rd, North

Chicago, IL 60064, USA
} 


\section{Background}

\section{Photosensitizers (PS)}

These are ring compounds whose soluble form is lightexcitable to produce cytotoxic reactive oxygen species (ROS) [1]. Naturally occurring PS include tetrapyrroles, e.g. corrins, chlorins and porphyrins - intermediates in the biosynthesis of vitamin B12, chlorophyll and heme [2]. The stoichiometry of these intermediates is stringently regulated by necessity to minimize their phototoxicity. Many plants produce PS as secondary metabolites for selfprotection, e.g. psoralen and hypericin [3]. Other PS are chemically synthesized: the fluorescein analogues, rose bengal and cyanosine, and phthalocyanines (PC). Natural and synthetic PS include Food and Drug Administration (FDA)-approved drugs, cosmetic, food and fabric dyes.

\section{PDT-generated singlet oxygen $\left({ }^{1} \mathrm{O}_{2}\right)$ and -cell susceptibility}

PDT has been used to eliminate tumors, pathogens and pests with cytotoxic ROS that is produced by illumination of targets treated with PS, e.g. porphyrins, PC and rose bengal, at their respective excitation wavelengths [4]. PDT initially generates singlet oxygen $\left({ }^{1} \mathrm{O}_{2}\right)$ and/or hydroxyl radicals, leading to the production of additional ROS, including peroxides and superoxides. ${ }^{1} \mathrm{O}_{2}$ is highly reactive and destructive, but too short-lived $(2-3 \mu \mathrm{s})$ to cross the cell membrane. ${ }^{1} \mathrm{O}_{2}$ is produced by plants during photosynthesis, but not by non-photosynthetic mammals, insects and Leishmania. Cells from the latter group are thus most susceptible to oxidative damage by ${ }^{1} \mathrm{O}_{2}$ because they lack mechanisms of detoxification. ${ }^{1} \mathrm{O}_{2}$ has the potential for strategic deployment to inflict maximal destruction of specific cell types with minimal collateral damage.

PDT, especially using ${ }^{1} \mathrm{O}_{2}$ generating PS for nonphotosynthetic cells, is unlikely to select for resistance, since neither light nor PS alone is cytotoxic. Their use in combination produces ROS inactivating multiple targets, minimizing the likelihood of selecting resistant traits. In support of this concept, no resistant Leishmania were selected after six consecutive cycles of PDT, i.e. induced uroporphyrinogensis plus light (see below) [5, 6]. Few survivors emerged after each PDT cycle as aporphyric cells, resulting from reduced uptake of the inducer and/ or heightened efflux of uroporphyrin I (URO). These phenotypes are not stable traits, since populations from the survivors after each of the six PDT cycles remain equally sensitive to the same PDT. Total inactivation of Leishmania by PDT is achievable when using two different PS, i.e. URO and PC (see below).

\section{Cellular uptake and subcellular targeting of PS for effective PDT}

The effectiveness of PDT is a function of light intensity delivered at a wavelength specific to the PS and its quantum yield [4]. Under physiological conditions, PDT is critically dependent on the uptake of PS by the target cells. The best example to illustrate this is the all-ornone phototoxicity of the ${ }^{1} \mathrm{O}_{2}$ generating URO, depending on its presence in the cytosol or in the extracellular milieu [5, 6]. URO is highly water-soluble, but not taken up by cells, like Leishmania. These cells are thus lightinsensitive and remain fully viable, as indicated by their active motility when bathed in URO-containing milieu [5]. This changes dramatically for uroporphyrinogenic Leishmania, which are transgenically modified to express the $2^{\text {nd }}$ and $3^{\text {rd }}$ enzymes in the heme biosynthetic pathway, rendering them inducible with the product of the $1^{\text {st }}$ enzyme in this pathway, i.e. delta-aminolevulinate (ALA) for cytosolic accumulation of URO [5-7]. During ALA-induced uroporphyrinogenesis, these mutants cease flagellar motility abruptly when examined under dim light for microscopy as URO begins to form in the cytosol $[5,6]$. Clearly, intracellular delivery of PS even in a minute amount is sufficient to sensitize cells to photoinactivation.

Cellular uptake of PS varies with their chemical structures. PC have been chemically modified to enhance such bioavailability. Modifications of their coordinating metals, side-chains and/or axial ligands increase cationicity for affinity to the negatively charged cell surface and solubility for persistence in the milieu $[8,9]$. Figure 1 shows some PS, which are localized to different subcellular sites of Leishmania. ALAinduced cytosolic accumulation of URO was discussed earlier (Fig. 1B, B'). The hydrophobic/lipophilic hypericin $\left(\mathrm{A}, \mathrm{A}^{\prime}\right)$ and aluminum phthalocyanine (Al-PC) (C, $\left.C^{\prime}\right)$ are taken up rapidly. These PS become associated immediately with and remain bound constantly to cellular membranes with undiminished fluorescence, but are transferrable from sensitized cells to the membranes of untreated cells [10]. How these phenomena are related to the expected turnover of cellular membranes is a question of interest for investigation. In contrast, the amino-PC [9] is endocytosed by Leishmania into their endosome-lysosome vacuolar system [11]. Other Sior $\mathrm{Zn}-\mathrm{PC}$ analogues [8] are either not taken up at all by Leishmania or are taken into the endosome-lysosome system or mitochondria [12].

Illumination of the PC-sensitized Leishmania with red light ( $\sim 600 \mathrm{~nm}$ excitation wavelength) at low fluence $\left(1-2 \mathrm{~J} \mathrm{~cm}^{2}\right)$ generates enough ${ }^{1} \mathrm{O}_{2}$ to inactivate them $[11,12]$. The inactivated cells lose their flagellar motility and viability, but remain intact structurally for hours before disintegration. In many instances, Leishmania differ from mammalian cells in their response to different PS for PDT. Elucidation of these differential mechanisms is of interest for optimizing the utility of PS for targeted PDT. 

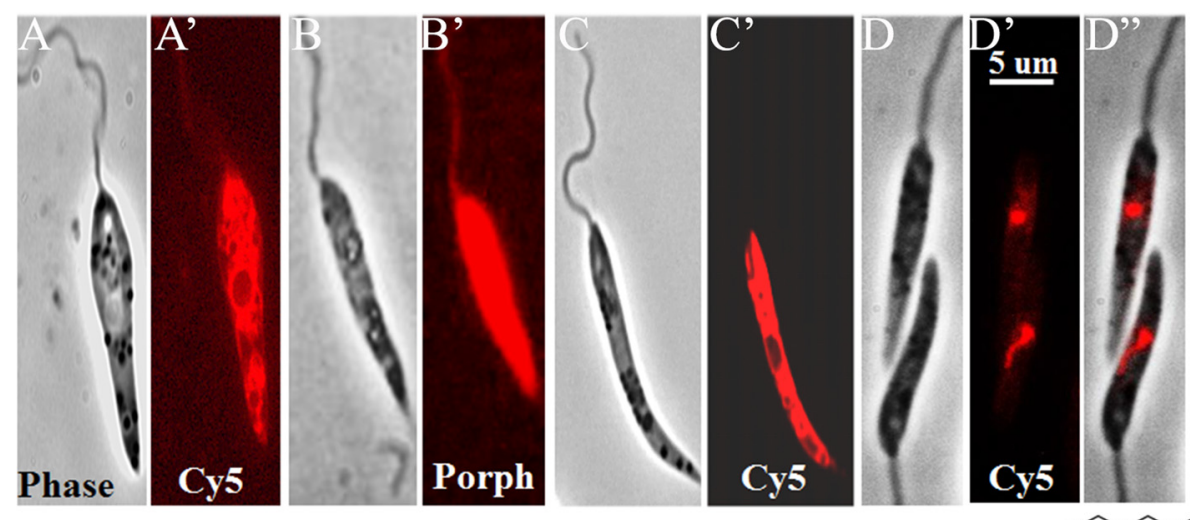

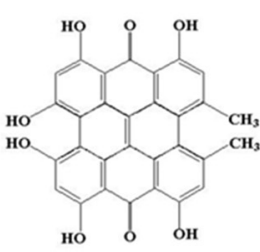

Hypericin

Membrane

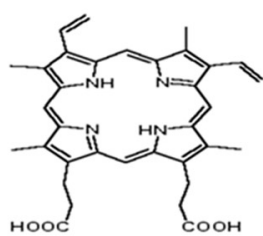

Porphyrin

Cytosolic

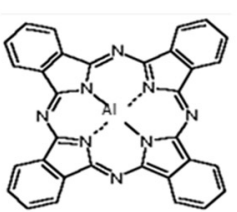

Al-

Phthalocyanine

Membrane

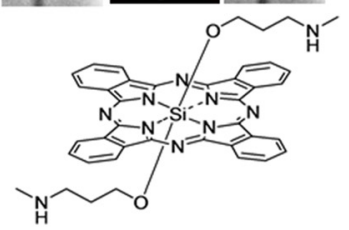

Amino-

Fig. 1 Photosensitization of Leishmania promastigotes with different photosensitizers. A-D, Phase contrast; A'-D", Fluorescence images taken under Cy5 or porphyrin filters. Under each image are the name, structure and cellular localization of the photosensitizer used. Cells were exposed to each photosensitizer overnight in the dark and imaged under live conditions as previously described [6, 7, 10-12]

PDT in clinical use: PDT of cutaneous leishmaniasis (CL) and post-treatment immune clearance of infection

PDT is an accepted clinical regimen for treating solid tumors and skin diseases, and for removing diseased tissues [4]. PDT begins with PS-sensitization of the target tissues with a PS or an inducer of endogenous PS, i.e. ALA to transiently up-regulate cellular porphyrin biosynthesis. The sensitized target is then illuminated to generate ROS for its destruction. Clinical PDT is thus limited to superficial and localized targets, e.g. solid tumors accessible to PS-sensitization and to the subsequent photo-inactivation by illumination from an external light source. Targets several centimeters below the skin are still PDT-treatable by using PC excitable with deeppenetrating red light.

PDT has been explored for treating infectious diseases of the skin [1], including cutaneous leishmaniasis (CL). Various PS have been assessed for PDT of experimental and clinical CL using different light sources: LED, laser and sun light (see [11]). PDT has the potential to shorten the often protracted duration of simple CL before spontaneous healing. The ultimate cure of all infectious diseases is thought to depend on post-therapeutic immune clearance, since no drug is expected to reach all individual pathogens in any given infection, regardless of the dosages used and the frequency of applications. The "post-PDT immune clearance" of CL foretells the potential of photodynamic vaccination (PDV) for both immunoprophylaxis and -therapy.

\section{Photodynamic vaccination (PDV)}

Prophylactic vaccination is the best preventive measure against infectious diseases, especially zoonosis, which cannot be controlled readily because of its persistence in animal reservoirs (Cf. [13]). Here we describe PDV using PDT-inactivation of Leishmania for vaccination. The evolution of Leishmania for intra-antigen-presenting cells (APC) parasitism and their sensitivity to PDT via PS accumulation are exploited for developing strategies to optimize the efficacy and safety of PDV.

\section{PDT-inactivation of Leishmania for vaccination against leishmaniasis \\ Background}

Lasting immunity after cure of leishmaniasis and "leishmanization" Development of effective prophylactic vaccines for this disease has long been considered as feasible from the lasting or life-long immunity seen after spontaneous healing of simple CL and after chemotherapeutic cure of visceral leishmaniasis (VL) (Cf. [14]). Infection of healthy individuals with lesion-derived live parasites in a hidden place is the crudest form of vaccination for simple CL. This is known as "leishmanization" [15] and has been practiced for millennia in the endemic 
sites of the Middle East and Central Asia. The vaccinees develop lasting immunity after self-healing and are thus immune for life from the potentially facial disfiguring $\mathrm{CL}$. The lasting immunity results from a $\mathrm{T}$ cell-mediated response to Leishmania naturally occurring vaccines, adjuvants and other immune-stimulating factors. The residence of Leishmania in APC makes these molecules readily available for processing and presentation, accounting very likely for the effective elicitation of cell-mediated immunity and the post-therapeutic immune clearance.

Leishmania vaccine availability, efficacy and safety Vaccines are still under development for both human and canine leishmaniasis. "Leishmanization" is effective, but unacceptable unless accomplished without a full-blown leishmaniasis. The extensive literature on the use of cultured Leishmania as the vaccine sources has been exhaustively reviewed recently (see Supplemental Table 1 in [16]). Live vaccines using avirulent strains, drug-crippled parasites and genetically attenuated mutants have been examined in experimental animal models. Most extensively studied are inanimate vaccines from the following materials: (1) whole-cells of cultured Leishmania killed or inactivated by chemical or physical means, e.g. formalinization, heating/autoclaving and irradiation; (2) soluble or insoluble fractions of cultured Leishmania or their secretory products; and (3) recombinant products of immunologically active Leishmania antigens. Prophylactic efficacy has been shown for most of them against experimental leishmaniasis in animal models, but few have reached the stages of clinical trials. Of note from these trials are the findings that inanimate vaccines from categories (2) and (3) are safe and immunogenic [17-19], but are only partially effective at best against human and canine leishmaniasis. The only whole-cell vaccine examined in category (1) is ineffective, but proven safe, i.e. autoclaved promastigotes at a dose of $\sim 200 \mathrm{ug}$ (100-400 ug) ([20]; F. Modabber, personal communication). This dosage is equivalent to $\sim 4 \times 10^{7}$ promastigotes, comparable to the number used as leishmanin (up to $2 \times 10^{7}$ promastigotes/ dose in phenol or merthiolate) in Montenegro skin test for delayed type hypersensitivity (DTH) [21]. These chemically or physically inactivated promastigotes have been injected into several hundred thousands of people. The continuing use of leishmanin test for DTH attests to the safety of whole-cell Leishmania when inactivated appropriately. Here we exploit PDT as a new modality of Leishmania inactivation for assessing the safety and efficacy of their use for vaccination.

\section{Three schemes of PDT-inactivated Leishmaina for vaccination}

The application of PDT in two steps (PS-sensitization followed by photo-inactivation) offers three different ways to inactivate Leishmania for vaccination, as depicted diagramatically in Fig. 2.

Scheme 1 uses the uroporphyrinogenic Leishmania transfectants [5-7], which have the wildtype efficiency for entry into APC and differentiation/replication in their phagolysosomes [22] (Events 1-4). The $1^{\text {st }}$ PDT step is the addition of ALA to the infected APC, resulting in porphyrinogenesis of both the intra-phagolysosomal Leishmania transfectants and their host APC (Event 5). The latter become aporphyric shortly afterward, since they possess a complete heme biosynthetic pathway, thereby rapidly exhausting the excessive porphyrins produced; In contrast, the transgenic Leishmania produce URO, which persists and accumulates in their cytosol because of their deficient heme biosynthesis pathway, lacking the downstream URO-utilizing enzymes (Event 6). Light-exposure of these infected APC excites URO in the uroporphyric Leishmania for their selective inactivation (Event 7) and eventual lysis to release antigens into the phagolysosomes and cytosol of the viable host APC (Event 8).

Scheme 2 is similar to Scheme 1, except that the uroporphyrinogenic Leishmania are doubly pre-PS-sensitized for the $1^{\text {st }}$ PDT step with ALA for URO accumulation in the cytosol and Si-PC for uptake into endosomes [11, 12]. These doubly PS-sensitized Leishmania infect APC in the dark, as described for Scheme 1 (Events 1-4). Subsequent light-exposure of these infected cells for the step 2 PDT produces the same outcome (Event 6), also as described for Scheme 1, except that the changes in the protocol reduce the events to 6 from 8 in Scheme 1.

Scheme 3 is similar to Schemes 1-2, except that uroporphyrinogenic Leishmania are doubly PS-sensitized and photo-inactivated to complete both PDT steps as described for Scheme 2 before use for loading APC (Event 1). The changes of the protocol simplify the events to 4 from 6 to 8 for schemes $1-2$. This scheme of APC-loading involves no replicative cycle of Leishmania in the host APC (Events 2-4).

\section{Cell-mediated immunity depicted for PDT vaccination}

Diagrammatic illustration Figure 3 depicts the elicitation of cell-mediated immunity by all three PDV schemes based on experimental evidence described in the subsequent paragraphs. PDT selectively inactivates intracellular Leishmania, resulting in the eventual release of their contents into the viable host APC (Event 1). The materials released from photolysed Leishmania are expected to include antigenic vaccines and other putative immune stimulating factors, as depicted in the foregoing sections. Several pertinent issues are of interest to mention here. APC in schemes 1-2 remain unscathed and viable after PDT [22]. This is expected, since these host APC are not PS-sensitized at the time of illumination, and since the ${ }^{1} \mathrm{O}_{2}$ produced is limited to 


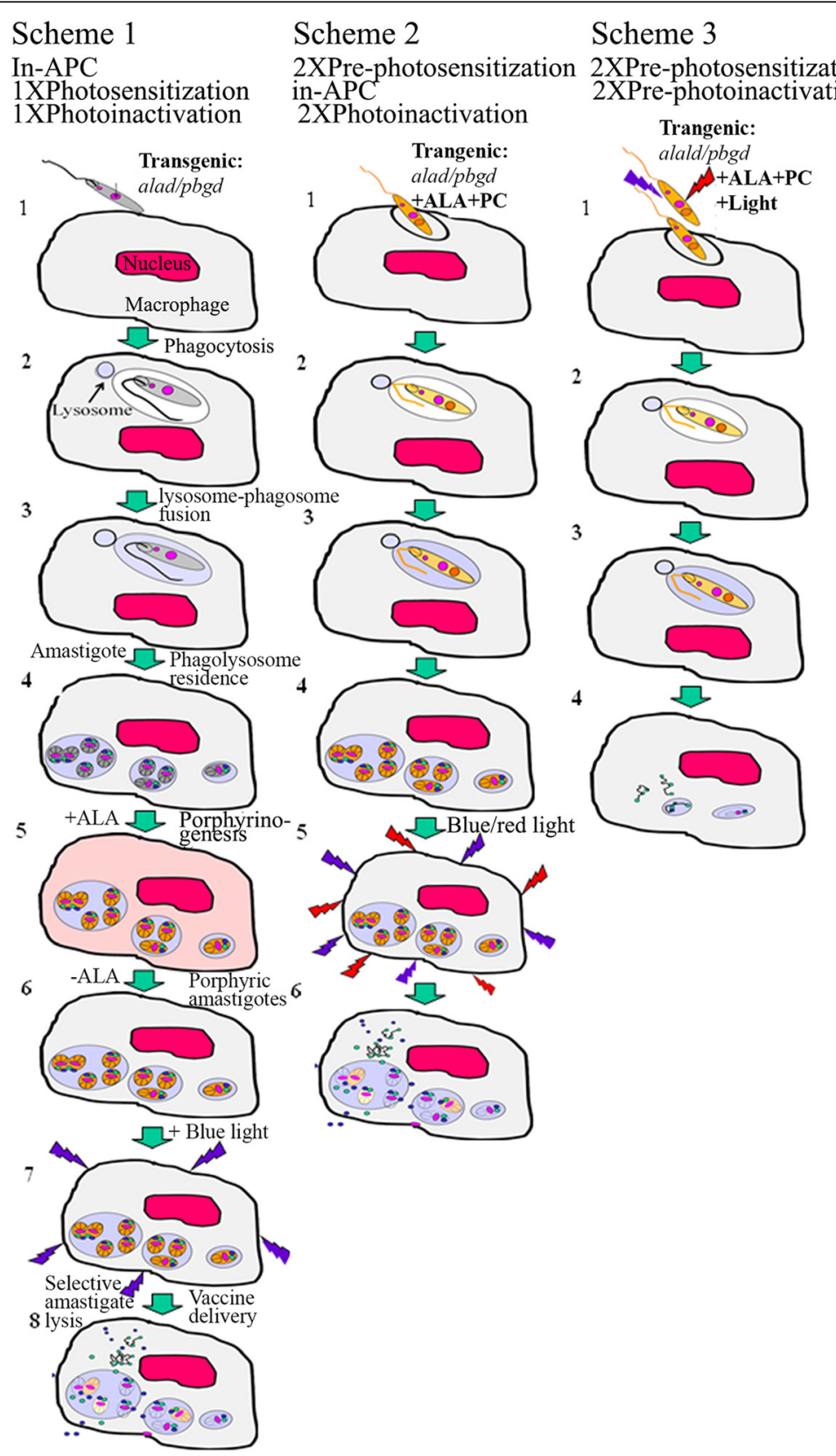

Fig. 2 Diagramatic illustration depicting three different schemes of Leishmania-based photodynamic vaccination in vitro. Transgenic:alad/pbgd, Porphyrinogenic Leishmania transfected with two mammalian cDNAs encoding the $2^{\text {nd }}$ and $3^{\text {rd }}$ enzymes in heme biosynthetic pathway, rendering them susceptible to delta-aminolevulinate (ALA)-induced neogenesis of uroporphyrin (URO); PC, Si-phthalocyanine photosensitizer $[6,11,12]$; Light, Illumination; Blue and red lightening symbols, Blue (400-500 nm wavelength) and red ( 600 nm wavelength) for excitation of URO and PC, respectively. Scheme 1: In-antigen presenting cell (APC) single PS-sensitization/photo-inactivation [22]. 1-2, Phagocytosis of porphyrinogenic, but untreated Leishmania by APC; 3, Fusion of Leishmania-containing phagosome with lysosome; 4, Leishmania differentiation into amastigotes and their replication in the phagolysosomes; 5, Exposure of the parasitized APC to ALA, resulting in porphyrinogenesis of both APC and phagolysosomal amastigotes; 6 , Removal of ALA, resulting in disappearance of porphyrins from APC and persistence of URO in amastigotes; 7-8, Illumination of these APC resulting in selective lysis of URO-loaded amastigotes, releasing vaccines into phagolysosomes and cytosol. Scheme 2: Same as Scheme 1, except that porphyrinogenic Leishmania are doubly PS-sensitized with ALA and PC in the dark before use for infecting APC [35]. 1-4, as described for Scheme 1, except that the Leishmania are pre-loaded with URO and PC, hence no further ALA treatment; 5-6, Illumination of the infected cells with blue and red light to excite URO and PC, lysing amastigotes with singlet oxygen and other ROS generated for releasing vaccines in APC. Scheme 3: Same as Schemes 1-2, except that Leishmania are pre-PS-sensitized and pre-photo-inactivated before use for vaccine delivery to APC [12]. 1-4, Uptake of oxidatively photo-inactivated Leishmania by APC, lysosome-phagosome fusion and their lysis to release vaccines as described 


\section{Processing/presentation of Leishmania-delivered vaccines}

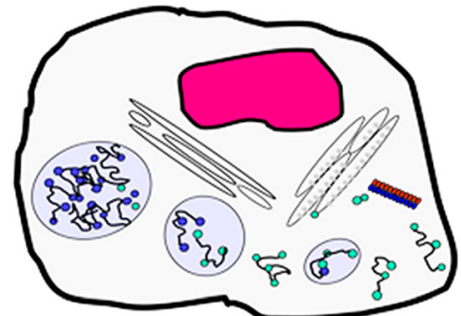

\section{Lysosomal pathway}

3. Proteosomal pathway
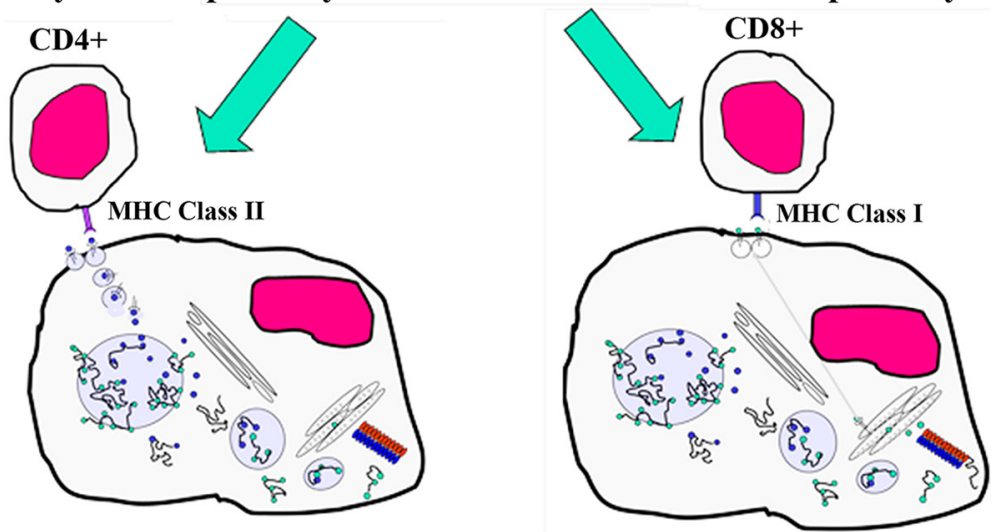

CD4+

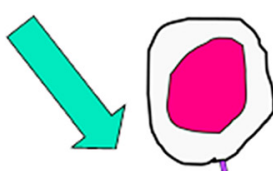

MHC Class II ?

CD8+

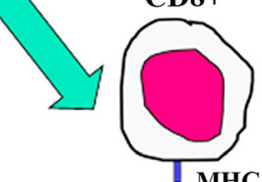


may further participate in the step of co-stimulation (not shown).

Experimental evidence The cell-mediated immunity depicted (Figs. 2 and 3) is based on the experimental outcome from the PDV schemes carried out in different experimental models, as briefly summarized below:

Scheme 1 was applied to immunization of Syrian Golden hamsters, eliciting a Th1 response for prophylaxis against Indian kala-azar produced by challenges with virulent Leishmania donovani [25]. The vaccination produces lasting immunity, as shown by the analysis of hepatosplenomegaly, parasite loads and cytokine profiles. Significantly, the immunity is adoptively transferable by splenic $\mathrm{T}$ cells from immunized animals to naïve hamsters, indicating that the immunity is cell-mediated and requires no antigen stimulation from persistent parasites, if any, at least in the recipients.

Scheme 2 was used for immunization of BALB/c mice against CL produced by challenges with Leishmania amazonensis. The observed prophylactic protection is significant, albeit incomplete, as indicated by comparing immunized mice versus the control groups. Immunization delayed the emergence of lesions for several weeks and significantly reduced the lesion size and their parasite loads by 10-fold versus the controls (Unpublished data. See legend to Fig. 4, Experimental-in-brief). The vaccination is considered effective, considering that BALB/C mice are known to bias toward Th2 with extreme levels of genetic susceptibility to cutaneous leishmaniasis.

Scheme 3 PDV used PDT-inactivated Leishmania, which were transfected to express ovalbumin (OVA) as a marker antigen or surrogate vaccine [12]. The cellmediated immune responses to OVA delivered by PDTinactivated transfectants were examined in in vitro and in vivo mouse models. APC loaded with the PDTinactivated Leishmania were shown to deliver OVA, which was effectively processed for MHC Class I presentation of its specific peptide for activation of CD8 $+\mathrm{T}$ cell line [12]. In the in vivo studies, BL57 mice were immunized three times, each with $\sim 10^{6}$ PDT-inactivated OVA-Leishmania. Splenic T cells of these immunized mice were activated in response to CD4+ and CD8+ T cell-specific OVA peptides that increased proportionally with the number of immunizations (Unpublished data. See legend to Fig. 4, Experimental-in-brief). Most significantly, $\mathrm{T}$ cell activation is 6-fold higher with OVA delivered by PDT-inactivated Leishmania than that delivered by conventional means.

The safety of Leishmania PDT-inactivation for vaccination increases in the order of Schemes 1 to 3. Leishmania were singly and doubly PDT-inactivated for Scheme 1 and Schemes $2-3$, respectively. They were completely inactivated by both PDT steps of PS-sensitization followed by double photo-inactivation before loading APC in Scheme 3 (see Fig. 4 and text for further discussion).

\section{PDT-inactivation of Leishmania for vaccine delivery against other infectious and malignant diseases}

The utility of PDT-inactivated Leishmania for delivery of add-on vaccines against other diseases is feasible, as indicated by the favourable outcome of the immune responses seen in vitro and in vivo to OVA delivered by this means. The successful delivery of OVA is significant, considering its expression at minuscule amount against a background of Leishmania proteins in overwhelming quantity and diversity in $\sim 10^{6}$ cells used for the delivery. This is taken to indicate that Leishmania creates no antigen-overload for vaccine delivery at least for OVA as a well-known $\mathrm{T}$ cell antigen.

Leishmania are naturally endowed with favourable attributes, making these parasites highly deployable as a universal vaccine carrier [22]. Many Leishmania species can be cultured safely as promastigotes in serum-free, chemically defined media [26] and scaled up for expansion [27]. The biosynthetic machineries of Leishmania are capable of high capacity transcription, translation and correct post-translational modification of foreign proteins. A number of efficient vectors are available for their abundant expression episomally or chromosomally as add-on vaccines in Leishmania - a favourable milieu of adjuvanticity and antigenicity conducive to elicit cellmediated immunity.

Efficient delivery of add-on vaccines by Leishmania is due to their surface coat, consisting of unique lipidsaccharide-protein complexes [28]. In natural infection, they are known to protect Leishmania against the lytic humeral factors abundant in the animal body fluids and to target them to the phagolysosomes of APC. This mode of parasitism is further facilitated by the secretory products of Leishmania, e.g. nucleoside diphosphate kinase [29]. Full deployment of these molecular attributes by Leishmania is expected to protect the payload of add-on vaccines for homing to APC when using non-sensitized or PS-sensitized Leishmania for vaccine delivery according to Schemes 1-2 (Fig. 2). Notably, Leishmania PDT-inactivated according to Scheme 3 are no longer viable, but remain OVA-delivery competent. The integrity of their surface coat may account for this, since it is unaffected by the ${ }^{1} \mathrm{O}_{2}$, which is generated in and limited to the cytosol of PDT-inactivated Leishmania.

Uroporphyrinogenic Leishmania are being evaluated for their ability to serve as a carrier of candidate vaccines for trials against other infectious and malignant diseases [30-33]. PDV with PDT-inactivated Leishmania transfectants will follow Schemes 1-3 (Fig. 2) to obtain safety and efficacy data. In vitro vaccination of DCs will 


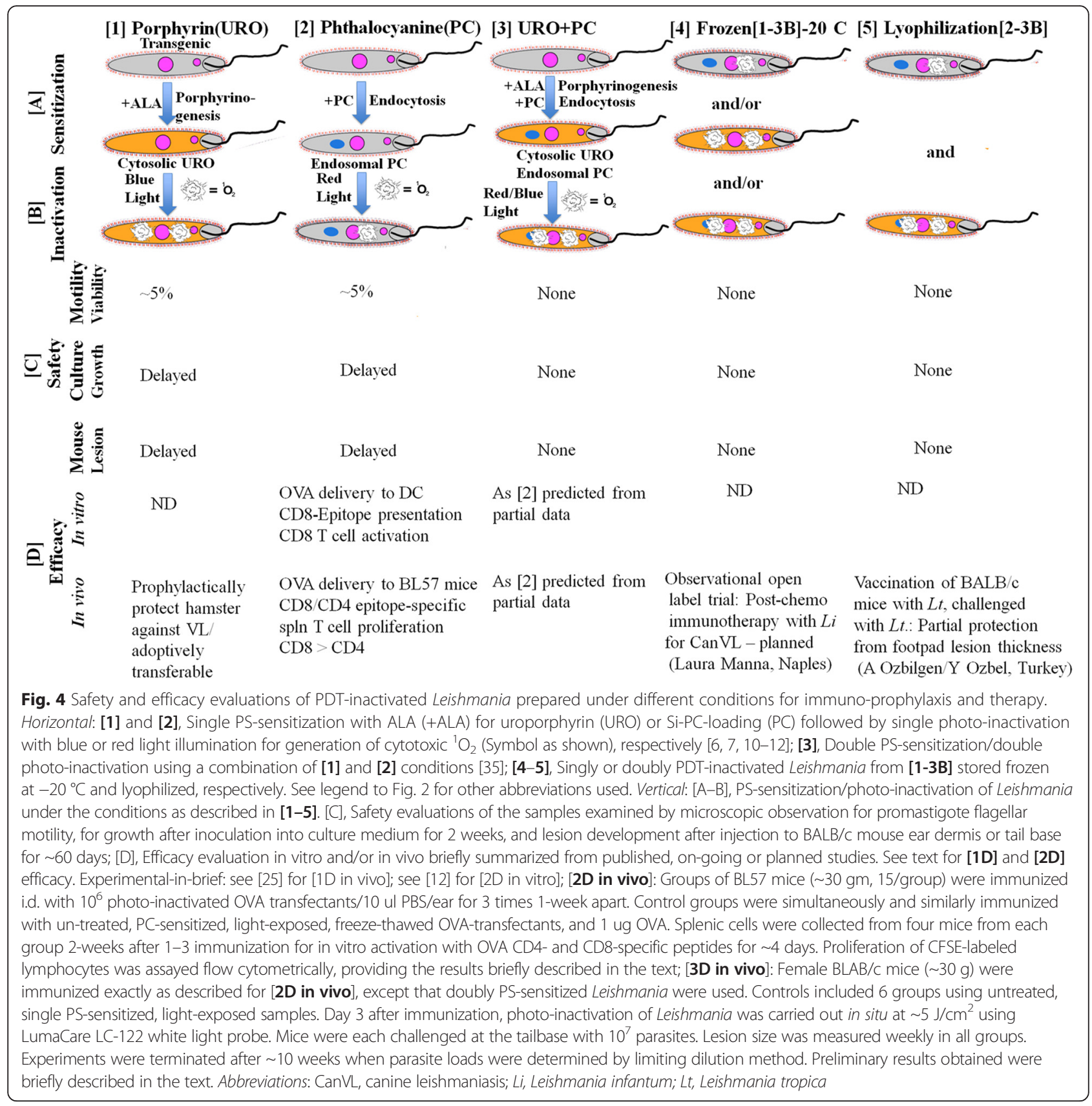

be pursued, as described [33, 34]. This presents a new approach by using a eukaryotic vehicle for safe and effective vaccine delivery.

\section{Safety versus efficacy evaluation of five Leishmania PDT-inactivation formats}

Figure 4 summaries the available data of Leishmania, which are PS-sensitized [A] and photo-inactivated [B] with or without additional treatments in different ways [1]-[5] for assessing their safety [C] and efficacy [D]. The safety is assessed after PDT inactivation of Leishmania by three different ways: microscopy for flagellar motility, cultivation for growth (2 weeks) and inoculation of mouse ear or tail base for lesion development ( $\sim 2$ months). Not all preparations were assessed by all criteria mentioned and the assessments for some samples are on-going or planned. The available results are briefly discussed below:

1. Single PDT of Leishmania by ALA-induced uropoprhyrinogensis [1] or PC-loading [2] alone inactivated $\sim 95 \%$ of these cells, as determined by the criteria described $[\mathrm{C}]$. Interestingly, PDV based on protocol [1] elicited adoptively transferable cell-mediated 
immunity and produced no visible pathology of the vaccination sites in hamster [25].

2. Double-PDT of Leishmania with a combination of Protocols 1-2 [3] resulted in no viable cells, as assessed by all three criteria $[C]$, indicative of a complete inactivation [35]. Immunization of BALB/C mice according to [3] is protective, although incomplete due to their inherent sensitivity to CL, as already discussed.

Products [4] and [5] prepared by freezing and lyophilization of PDT-inactivated Leishmania [1-3B], respectively, were undertaken to facilitate their storage and transport and to increase their safety at the expense of their efficacy. Although still on-going, lyophilized samples [5] appear to have some prophylactic activities against $\mathrm{CL}$ challenges after immunization of $\mathrm{BALB} / \mathrm{c}$ mice.

From the available data, the double-PDT inactivation of Leishmania by method [3] provides the best vaccination format for use with optimal safety and efficacy. The other regimens are being optimized for further safety versus efficacy evaluation.

\section{Photodynamic insecticides (PDI) \\ Background}

History PDT to control insect pests was first mentioned in the early 1900's (see [36]). From 1980's to1990's, The American Chemical Society published several symposium volumes on "Light-activated pesticides" [37-39]. Since then, follow-up publications have been limited and were summarized in the reviews [36, 40, 41]. Different dyes were used in experimental and/or field trials as PDI against various insects, mainly mosquito larvae and Mediterranean fruit flies. Industrial interests (PhotoDye International, Inc) included aerial spray of dye mixtures (xanthenes) or "SureDye" (Red Dye \#28 and Yellow Dye \#8) (http://www.cdpr.ca.gov/docs/emon/pubs/ehapreps/ suredye.htm) in attempt to control the latter pest. The work in the past decades showed some effectiveness of PDI, but this area of research has not gained attention.

Preamble PDI has the potential as an effective measure to control disease-transmitting vectors and other harmful insects. Development of resistance by insect pests to insecticides is a recurrent scenario [42], calling attention to different approaches, like PDT, which is unlikely to elicit resistance. The potential of PDI to control different insect pests are briefly discussed below.

Phytophagous insects cause substantial losses in crops and livestock despite the use of genetically modified (GM) insect-resistant plants [43]. Phloem/xylem sapfeeding insects cause additional damage by transmitting plant diseases. These vectors are PDT-targetable, since they engorge voluminous plant saps amenable to PSloading and are translucent to light for photo-inactivation. The use of ${ }^{1} \mathrm{O}_{2}$-generating PS for PDT has the potential to discriminate these and other phytophagous insects for selective killing, sparing their photosynthetic and ${ }^{1} \mathrm{O}_{2}$-resistant host plants.

Many animal biting insects feed on blood and transmit serious diseases, accounting for substantial morbidity and mortality of domestic animal and human populations worldwide. Application of PDI to control such insect vectors is highly desirable, e.g. Anopheles mosquitoes, which transmit malaria and Aedes spp., which transmit Chikungunya, Dengue and Zika fever, causing epidemics in the tropical/subtropical world today. The only new non-PDI approach to control these vectors is to release GM mosquitoes based on Wolbachia- or male-induced infertility $[44,45]$. For PDT of female mosquitoes and other blood feeders (phototropic and day-light active species), PS is deliverable via the bloodstream of susceptible hosts or the use of suitable baits to sensitize the insects for sun light inactivation. The larval stages of all mosquitoes (and also black flies) are aquatic and thus are receptive to water-soluble PS for PDT [46, 47].

PS-sensitization of all insects is possible by direct spraying for their uptake via surface contact and/or systematically via the hosts, as used for the current insecticides. Direct incorporation of PS into the drinking and food sources of insects will deliver them into the digestive tracts for sensitization of cells therein. In either case, accessibility of PS-sensitized cells to light is necessary to generate cytotoxic ROS for target destruction. Nocturnal and darkness-loving insects are less amenable to PDT unless a light-emitter is provided with the PS for their excitation.

Summarized below are some observations from our preliminary studies of few insects on their uptake of selected PS and susceptibility to PDI.

\section{Screening of PS for their PDI against selected insects}

Exposure of the $4^{\text {th }}$ instar mosquito larvae (Culex pipiens quinquefasciatus) and adult sand flies (Phlebotomus dubosqi) [48] to rose bengal (RB) and cyanosine (CY) overnight resulted in the accumulation of these red dyes that are visible in the gut of the larvae (Fig. 5a) and of both female and male flies (Fig. 5b, c). Their uptake of the other PS examined is less clear, including aluminumphthalocyanine (Al-PC), protoporphyrin IX (PROTO) and Nile blue sulfate (NB). Only RB- and CY-sensitized larvae lost their viability after light exposure based on their mobility (not shown). The sand fly response to the PDI is inconclusive due to a high mortality of the control group, pending further investigation. This is also true for PDI of the plant-sucking insects, e.g. aphids, suggestive of a need 


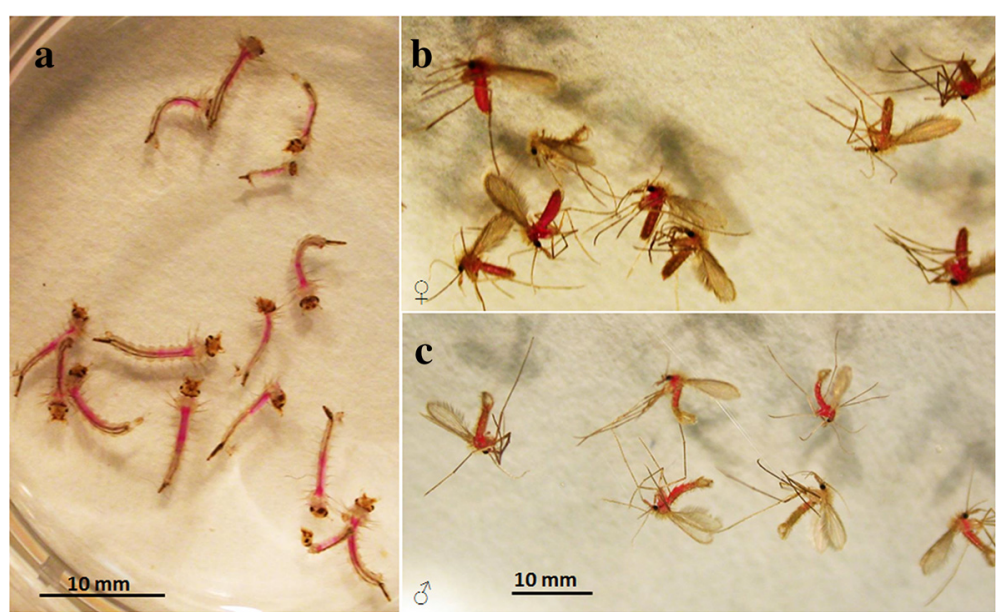

Fig. 5 Uptake of rose bengal by selected insects and their photo-inactivation. a Culex pipiens quinquefasciatus 4th instar larvae exposed to rose Bengal $(10 \mathrm{ug} / \mathrm{ml})(\sim 20$ larvae $/ 5 \mathrm{ml}$ water/well in 6-well plate) in dark for $\sim 24$ hours followed by exposure to white light for $\sim 6 \mathrm{~h}$ at $\sim 2500$ lux; b-c Phlebotomus duboscqi female (b) and male (c) adult flies ( 20 flies/screened paper cup) fed with $5 \%$ sucrose solution and $500 \mathrm{ul}$ of $5 \mathrm{mg} / \mathrm{ml}$ rose bengal in a cotton ball for $\sim 20 \mathrm{~h}$ in the dark followed by exposure to $\sim 2500$ lux of white light for $3 \mathrm{~h}$. Duplicate samples were prepared and kept in the dark as controls. Rose bengal is taken up by the flies of both sexes. Phototoxicity is evident for the mosquito larvae, but inconclusive for the flies. The tests were done in Petr Volf's lab

to use membrane-feeding techniques instead of using cut or potted plants $[49,50]$.

Our observations as described are preliminary, but represent the first study of PDI on sand flies, showing their uptake of PS used. The mosquito larvicidal activities of the PDI seen are consistent with the results of an early work (see [36]) and the reports using marigold alpha-terthienyl as the PS and different mosquito species [46, 47].

\section{Uptake of PS by mosquito cells in vitro}

Since the uptake of PS by mammalian and Leishmania cells is a prerequisite for their sensitization for PDT, we have begun to assess this with insect cells, e.g. Aedes albopictus clone C6/36 (ATCC CRL-1660). Figure 6 shows the uptake of RB and CY by these mosquito cells, rendering them sensitive to photo-inactivation. Untreated cells ([1]-None) are adherent (1A-DIF) and

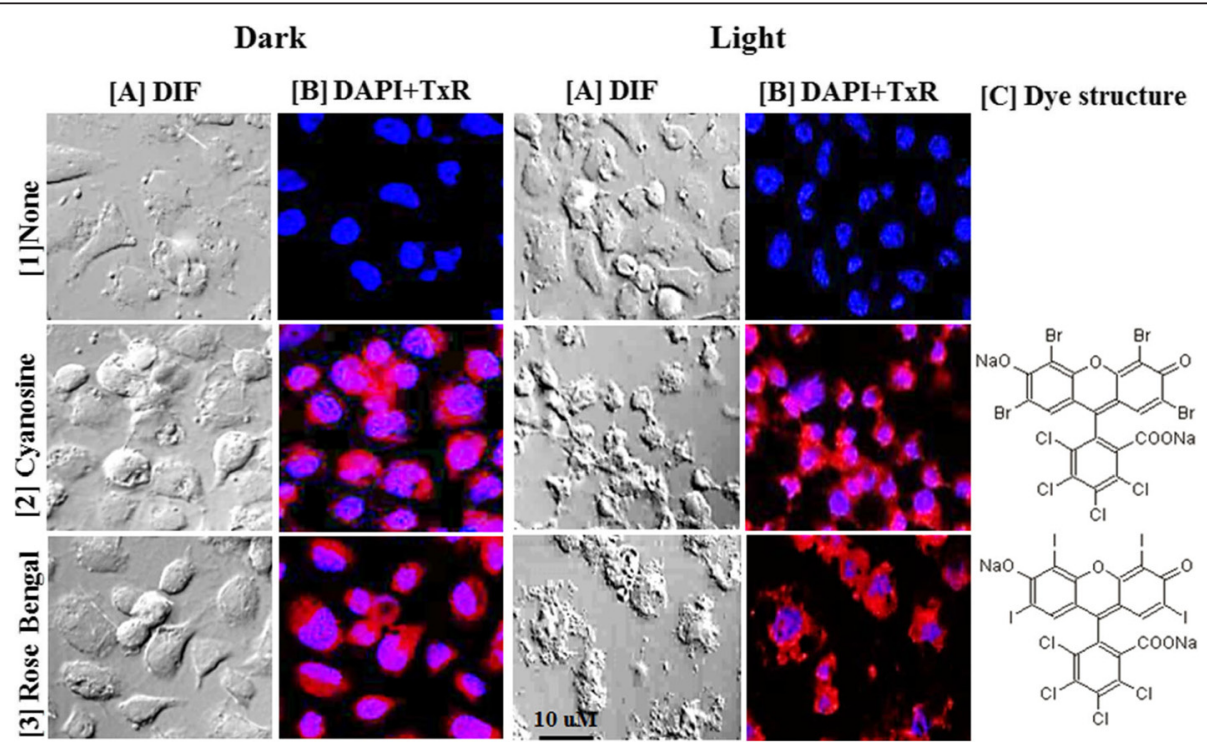

Fig. 6 Uptake of cyanosine and rose bengal by mosquito cells of the C6/36 line and their photosensitivity in vitro. The insect cells were exposed to both dyes overnight and illuminated with white light under conditions similar to those described for mammalian and Leishmania cells [10-12, 22]. Images were captured first under differential interference (DIF) [A] and then under the filter sets for DAPI and Texas red [B]. [C] Chemical structure of cyanosione and rose Bengal. Uptake of both dyes by the cells after incubation in the dark overnight (Dark, A2-3, B2-3) and cellular disintegration after light exposure for $4 \mathrm{~h}$ (Light, A2-3, B2-3) in contrast to the untreated controls (Dark and Light, A1, B1). Work done by Shin-Hong Shiao 
non-fluorescent (1B DAPI + TxR), irrespective of illumination (1 Dark and Light). Cells exposed to CY [2] and RB [3] show cytoplasmic fluorescence (2B, 3B DAPI-txR), indicative of dye uptake. Sensitized cells remain adherent and intact (Dark, 2A, 3A-DIF), but become disintegrated after light-exposure (Light, 2A, 3A-DIF). These results are consistent with the larvicidal activities of $\mathrm{RB}$ and $\mathrm{CY}$ observed, providing a cellular basis for their PDT activities. Notably, the mosquito cells were not sensitized for PDT with the following PS: Al-PC [10], PC3-4 [11], NB and a porphyrin analogue [51]. Insect cells are thus similar to other cells in their requirement of PS uptake for susceptibility to PDT, but require different PS for PDI.

The preliminary data point to the feasibility of screening additional PS for PDT of cells from different insects, both harmful and beneficial, and from other life forms in their environments. Such in vitro screening of PS for activities has the potential to identify PDI, which discriminate harmful pests from beneficial insects and other friendly organisms for selective killing of the former. Of further interest is to elucidate the mechanisms of differential PS-uptake by cells of different origin, providing clues for designing PS with specificity for PDI targeting.

\section{Conclusions}

PDT-inactivation of Leishmania offers the versatility and flexibility to balance safety versus efficacy for vaccination against leishmaniasis and as potential carriers of vaccines against other infectious and malignant diseases (PDV). The development of this new approach will benefit from governmental and public acceptance and support. The ingenuity of the new leadership [52] is needed for novel regulation that will ensure the safety of vaccines with no barrier to disrupt innovation. The advocacy groups also call attention to rectify the existing barriers between science and cures, e.g. fasterCures (http://www.fastercures.org/). Development of vaccines including PDV will further benefit from effective measures against the anti-vaccination movement [53].

PDI represents an alternative approach to control insect pests. It is still in its early infancy of development despite the idea first emerged almost 100 years ago. Many PS for PDI are innocuous compounds, which have long been used among our everyday household products. Their application as PDI is not expected to select for resistance in contrast to the chemical pesticides in current use. PDI has the potential to complement the GM approaches in the field of agriculture and medicine. It will be particularly suitable for development in places where the population is sensitive to GM organisms.

The lynchpin between PDV and PDI is the PS for light excitation to generate cytotoxic ROS. The expertise in medicinal chemistry is essential for synthesis and design of novel PS. This depends on the input of biologists to elucidate the mechanisms of their cellular/molecular activities. New PS need to be assessed by expert clinicians, veterinarians, entomologists, cancer researchers, microbiologists and immunologists, hence the consortium of collaborators enlisted.

\section{Abbreviations \\ ALA, delta-aminolevulinate; Al-PC, aluminum phthalycyanine; APC, antigen- presenting cells; $\mathrm{CL}$, cutaneous leishmaniasis; $\mathrm{CY}$, cyanosine; DIF, differential interference; DTH, delayed type hypersensitivity; GMO, genetically modified organisms; NB, Nile blue; OVA, ovalbumin; PC, phthalocyanines; PDI, photodynamic insecticide; PDT, photodynamic therapy; PDV, photodynamic vaccination; PROTO, protoporphyrin IX; PS, photosensitizer; RB, rose bengal; $\mathrm{ROS}$, reactive oxygen species; URO, uroporphyrin I; VL, visceral leishmaniasis}

\section{Acknowledgements}

Thanks are due to Joseph Reynolds and David Everly for reviewing this manuscript. Publication of the CVBD 11 thematic series has been sponsored by Bayer HealthCare - Animal Health division.

\section{Collaboration group}

Ramesh B. Batchu, PhD, Director, Division of Surgical Oncology \&

Developmental, Therapeutics, Associate Professor, The Michael and Marian Ilitch Department of Surgery, Wayne State University, 4646 John R Road, Detroit, MI 48201, USA. rameshbabu.batchu@gmail.com

Hui-Wen (Winni) Chen, DVM, PhD (陳慧文), Assistant Professor, Department of Veterinary Medicine, National Taiwan University, 1 Sec. 4 Roosevelt Rd., Taipei, 10617 Taiwan. winnichen@ntu.edu.tw Larry Ming C. Chow, ScD (周銘祥), Associate Head and Professor, Department of Applied Biology and Chemical Technology, Hong Kong Polytechnic University, Hong Kong. larry.chow@polyu.edu.hk

Robert Elliott, MD/Jonathan F. Head, PhD, Elliott Mastology Center, 541 Shadows Lane, Baton Rouge, LA 70806, USA. relliott@eehbreastca.com Chia-Kwung Fan PhD (范家㫃), Professor and Chairman, Department of Molecular Parasitology and Tropical Diseases, School of Medicine and Center for International Tropical Medicine, College of Medicine, 250 Wu-Xing Street, Taipei Medical University, Taipei, Taiwan. tedfan@tmu.edu.tw

Chen-Hsiung Hung, PhD (洪政雄), Investigator and Vice Director, Institute of Chemistry, Academia Sinica, 128, Sec. 2, Academia Road "Nankang, Taipei, Taiwan 11529. chhung@gate.sinica.edu.tw

Dar-Der Ji, PhD (嵇達德), Associate Professor, Department of Tropical Medicine/International Health Programs, National Yang-Ming University, Beitou, Taipei 112, Taiwan. ddji@ym.edu.tw

Zhao-Rong Lun, PhD (伦照荣), Professor \& Director, Center for Parasitic Organisms, School of Life Sciences, Sun Yat-Sen University, Guangzhou 510275, P.R. China. Isslzr@mail.sysu.edu.cn

Laura Manna, PhD, DVM, School of Veterinary Medicine and Animal Productions, University of Naples, Federico II, Naples, Italy. laumanna@unina.it Yoshitsugu Matsumoto, DVM, PhD, Professor, Laboratory of Molecular Immunology, School of Agricultural and Life Sciences, The University of Tokyo, Yayoi 1-1-1, Tokyo, Japan 113-8657 aysmatsu@mail.ecc.u-tokyo.ac.jp Dennis KP Ng, DPhil (吳基培), Professor, Department of Chemistry, Chinese University of Hong Kong, Sha Tin, N. T., Hong Kong. dkpn@cuhk.edu.hk Camila I. de Oliveira PhD/Sayonara Melo, Centro de Pesquisas Gonçalo Moniz. FIOCRUZ, R. Waldemar, Falcão, 121 Candeal, Salvador- BA -40296-710, Brazil.camila@bahia.fiocruz.br

Yusuf Ozbel, MD, Professor, Ege University Faculty of Medicine, Department of Parasitology, 35100 Bornova, Izmir,TURKEY. yusuf.ozbel@gmail.com Ahmet Özbilgin. PhD, Professor, Celal Bayar Üniversitesi, Tip Fakültesi Parazitoloji A.D, Dekanlik Binasi Uncubozköy, 45030, Manisa, Türkiye. a.ozbilgin@yahoo.com

Joseph Reynolds, PhD, Department of Microbiology/Immunology, Chicago Medical School/RFUMS, N Chicago, IL 60064, USA.

joseph.reynolds@rosalindfranklin.edu

Chizu Sanjoba, PhD, Laboratory of Molecular Immunology, Graduate School of Agricultural and Life Sciences, The University of Tokyo, 1-1-1Yayoi, Bunkyo-ku, Tokyo 113-8657 Japan. asanjoba@mail.ecc.u-tokyo.ac.jp 
Shin-Hong Shiao, PhD (蕭信宏), Assistant Professor, Department of Parasitology, Institute of Microbiology, School of Medicine, National Taiwan University, Taipei, Taiwan. shshiao@ntu.edu.tw Nang-Yao Shih, PhD (施能耀), Associate Investigator, National Institute of Cancer Research, National Health Research Institutes, Tainan, Taiwan. jshih@nhri.org.tw

Chi-Wei Tsai, PhD (蔡志偉), Associate Professor, Department of Entomology, College of Natural Resources and Agriculture, National Taiwan University, 1 Sec. 4 Roosevelt Rd., Taipei, 10617 Taiwan. chiwei@ntu.edu.tw Maria da Graça H. Vicente, PhD, Distinguished Professor, Department of Chemistry, 249 Chemistry and Materials Building, Highland Rd, Louisiana State University, Baton Rouge, LA 70803, USA. vicente@lsu.edu Petr Volf, PhD, Professor, Department of Parasitology, Faculty of Science, Charles University Vinicna 7, 12844 Praha 2, Czech Republic. volf@cesnet.cz Yueh-Lung Wu, PhD (吳岳隆), Assistant Professor, Department of Entomology, College of Natural Resources and Agriculture, National Taiwan University, 1 Sec. 4 Roosevelt Rd., Taipei, 10617 Taiwan. runwu@ntu.edu.tw Chao-Lan Yu, PhD (郁兆蘭), Professor, Department of Biomedical Sciences, Chang Gung University, No. 259, Wenhua 1st Rd., Guishan Dist., Taoyuan City 33302, Taiwan.clyu@mail.cgu.edu.tw

Xiao-Nong Zhou, PhD (周晓农),Professor and Director, National Institute of Parasitic Diseases, Chinese Center for Disease Control and Prevention, 207 Rui Jin Er Road, Shanghai 200025, P.R. China.zhouxn1@chinacdc.cn ipdzhouxn@sh163.net

\section{Funding}

Work described has received partial support by NIH/NIAID Grant \# Al097830, Al-7712375, Al-68835 and other sources.

\section{Availability of data and materials}

Not applicable.

\section{Authors' contributions}

KPC organized and wrote the first draft of this manuscript. BKK joined KPC to refine the science and language of the writing and the illustrations to complete the manuscript for submission. Both authors have read and approved the final version of the manuscript.

\section{Competing interests}

The authors declare that they have no competing interests.

\section{Consent for publication}

Not applicable.

Received: 1 April 2016 Accepted: 29 June 2016

Published online: 13 July 2016

\section{References}

1. Abrahamse H, Hamblin MR. New photosensitizers for photodynamic therapy. Biochem J. 2016:473:347-64.

2. Battersby AR. Tetrapyrroles: the pigments of life. Nat Prod Rep. 2000;17:507-26.

3. Berenbaum M. Phototoxicity of plant secondary metabolites: insect and mammalian perspectives. Arch Insect Biochem Physiol. 1995;29:119-34.

4. Benov L. Photodynamic therapy: current status and future directions. Med Princ Pract. 2015;24 Suppl 1:14-28.

5. Sah JF, Ito H, Kolli BK, Peterson DA, Sassa S, Chang KP. Genetic rescue of Leishmania deficiency in porphyrin biosynthesis creates mutants suitable for analysis of cellular events in uroporphyria and for photodynamic therapy. $J$ Biol Chem. 2002;277:14902-9.

6. Dutta S, Kolli BK, Tang A, Sassa S, Chang KP. Transgenic Leishmania model for delta-aminolevulinate-inducible monospecific uroporphyria: cytolytic phototoxicity initiated by singlet oxygen-mediated inactivation of proteins and its ablation by endosomal mobilization of cytosolic uroporphyrin. Eukaryot Cell. 2008;7:1146-57.

7. Dutta S, Furuyama K, Sassa S, Chang KP. Leishmania spp. deltaaminolevulinate-inducible neogenesis of porphyria by genetic complementation of incomplete heme biosynthesis pathway. Exp Parasitol. 2008;1 18:629-36.

8. Li H, Jensen TJ, Fronczek FR, Vicente MG. Syntheses and properties of a series of cationic water-soluble phthalocyanines. J Med Chem. 2008;51:502-11.
9. Jiang XJ, Yeung SL, Lo PC, Fong WP, Ng DKP. Phthalocyanine-polyamine conjugates as highly efficient photosensitizers for photodynamic therapy. J Med Chem. 2011;54:320-30.

10. Dutta S, Ray D, Kolli BK, Chang KP. Photodynamic sensitization of Leishmania amazonensis in both extracellular and intracellular stages with aluminum phthalocyanine chloride for photolysis in vitro. Antimicrob Agents Chemother. 2005;49:4474-84.

11. Al-Qahtani A, Alkahtani S, Kolli B, Tripathi P, Dutta S, Al-Kahtane AA, et al. Amino-phthalocyanine-mediated photodynamic inactivation of Leishmania tropica. Antimicrob Agents Chemother. 2016;60:2003-11.

12. Dutta S, Ongarora BG, Li H, Vicente MG, Kolli BK, Chang KP. Intracellular targeting specificity of novel phthalocyanines assessed in a host-parasite model for developing potential photodynamic medicine. PLoS One. 2011;6:e20786.

13. Chang KP. Vaccination for disease prevention and control: The necessity of renewed emphasis and new approaches. J Immunol Immunotech. 2014;1:1-4. doi:10.17653/2374-9105.SSe0001.

14. Chang KP. Leishmaniases. In: Brown M, editor. Encyclopedia of Life Sciences. New York: John Wiley \& Sons, Ltd.; 2012. doi:10.1002/9780470015902. a0001954.pub3.

15. Khalil EAG, El Hassan AM, Zijlstra EE, Mukhtar MM, Ghalib HW, Musa B, et al. Autoclaved L. major vaccine for prevention of visceral leishmaniasis: a randomised, double-blind, BCG-controlled trial in Sudan. Lancet. 2000;356:1565-9.

16. Costa CH, Peters NC, Maruyama SR, de Brito Jr EC, Santos IK. Vaccines for the leishmaniases: proposals for a research agenda. Working Group on Research Priorities for Development of Leishmaniasis Vaccines. PLoS Negl Trop Dis. 2011;5:e943.

17. Gradoni L. Canine Leishmania vaccines: still a long way to go. Vet Parasitol. 2015:208:94-100.

18. Chakravarty J, Kumar S, Trivedi S, Rai VK, Singh A, Ashman JA, et al. A clinical trial to evaluate the safety and immunogenicity of the LEISH-F1+MPL-SE vaccine for use in the prevention of visceral leishmaniasis. Vaccine. 2011;29:3531-7.

19. Llanos-Cuentas A, Calderón W, Cruz M, Ashman JA, Alves FP, Coler RN, et al. A clinical trial to evaluate the safety and immunogenicity of the LEISH-F1+MPL-SE vaccine when used in combination with sodium stibogluconate for the treatment of mucosal leishmaniasis. Vaccine. 2010;28:7427-35.

20. Kamil AA, Khalil EA, Musa AM, Modabber F, Mukhtar MM, Ibrahim ME, et al. Alum-precipitated autoclaved Leishmania major plus Bacille Calmette-Guérrin, a candidate vaccine for visceral leishmaniasis: safety, skin-delayed type hypersensitivity response and dose finding in healthy volunteers. Trans R Soc Trop Med Hyg. 2003;97:365-8.

21. Wilcocks C, Manson-Bahr PEC. Manson's tropical diseases. 17th ed. London: Bailiere-Tindall; 1976.

22. Dutta S, Chang C, Kolli BK, Sassa S, Yousef M, Showe M, et al. Deltaaminolevulinate-induced host-parasite porphyric disparity for selective photolysis of transgenic Leishmania in the phagolysosomes of mononuclear phagocytes: a potential novel platform for vaccine delivery. Eukaryot Cell. 2012;11:430-41.

23. Gracanin M, Hawkins CL, Pattison DI, Davies MJ. Singlet-oxygen-mediated amino acid and protein oxidation: formation of tryptophan peroxides and decomposition products. Free Radic Biol Med. 2009;47:92-102.

24. Kotsias F. Hoffmann E, Amigorena S, Savina A. Antioxid Reactive oxygen species production in the phagosome: impact on antigen presentation in dendritic cells. Redox Signal. 2013:8:714-29.

25. Kumari S, Samant M, Khare P, Misra P, Dutta S, Kolli BK, et al. Photodynamic vaccination of hamsters with inducible suicidal mutants of Leishmania amazonensis elicits immunity against visceral leishmaniasis. Eur J Immunol. 2009:39:178-91.

26. Chang KP, Hendricks DL. Laboratory cultivation and maintenance of Leishmania. In: Chang KP, Bray RS, editors. Human parasitic diseases, Leishmaniasis, vol. 1. Amsterdam: Elsevier; 1985. p. 213-46.

27. Fritsche C, Sitz M, Weiland N, Breitling R, Pohl HD. Characterization of the growth behavior of Leishmania tarentolae: a new expression system for recombinant proteins. J Basic Microbiol. 2007;47:384-93.

28. Chang KP, Reed SG, McGwire BS, Soong L. Leishmania model for microbial virulence: the relevance of parasite multiplication and pathoantigenicity. Acta Trop. 2003:85:375-90

29. Kolli BK, Kostal J, Zaborina O, Chakrabarty AM, Chang KP. Leishmaniareleased nucleoside diphosphate kinase prevents ATP-mediated cytolysis of macrophages. Mol Biochem Parasitol. 2008;158:163-75. 
30. Chen HW, Wang CH. A multiplex reverse transcriptase-PCR assay for the genotyping of avian infectious bronchitis viruses. Avian Dis. 2010;54:104-8.

31. Elliott RL, Head JF. Adjuvant breast cancer vaccine improves disease specific survival of breast cancer patients with depressed lymphocyte immunity. Surg Oncol. 2013;22:172-7.

32. Shih NY, Chang GC, Tseng SW, Liu K, Hsiao KC, Lin HC. Targeting to tumorassociated antigen eno 1 provides preventive and therapeutic effects on disease progression of lung cancer. Ann Oncol. 2013;24 Suppl 1:i27-9.

33. Batchu RB, Gruzdyn OV, Moreno-Bost AM, Szmania S, Jayandharan G, Srivastava A, et al. Efficient lysis of epithelial ovarian cancer cells by MAGE-A3-induced cytotoxic T lymphocytes using rAAV-6 capsid mutant vector. Vaccine. 2014;32:938-43.

34. Batchu RB, Gruzdyn O, Potti RB, Weaver DW, Gruber SA. MAGE-A3 with cell-penetrating domain as an efficient therapeutic cancer vaccine. JAMA Surg. 2014;149:451-7.

35. Dutta S, Waki K, Chang KP. Combinational sensitization of Leishmania with uroporphyrin and aluminum phthalocyanine synergistically enhances their photodynamic inactivation in vitro and in vivo. Photochem Photobiol. 2012;88:620-5.

36. Ben Amor T, Jori G. Sunlight-activated insecticides: historical background and mechanisms of phototoxic activity. Insect Biochem Mol Biol. 2000;30:915-25.

37. Heitz JR, Downum K. Light activated pest control. Am Chem Soc Symp Series. 1987;339. Doi: 10.1021/bk-1987-0339 (http://pubs.acs.org/isbn/ 9780841210264); Am Chem Soc Symp Series. 1995;616. (http://pubs.acs.org/ doi/pdf/10.1021/bk-1995-0616.fw001)

38. Duke SO, Rebeiz CA. Porphyric pesticides: chemistry, toxicology and pharmaceutical applications. Washington DC. Am Chem Soc Symp Series. 1994;559:317 (http://www.loc.gov/catdir/enhancements/fy0605/ 94016418-t.html).

39. Heitz JR. Pesticidal applications of halogenated xanthene dyes. Phytoparasitica Guest Editorial. 1997;25:89-92 (http://link.springer.com/ article/10.1007/BF02981188).

40. Rebeiz CA, Guta L, Leea K, Juvika JA, Rebeiza CC, Boutona CE, et al. Photodynamics of porphyric insecticides. Crit Rev Plant Sci. 1995;14:329-66.

41. Mangan RL, Moreno DS. Photoactive dye insecticide formulations: adjuvants increase toxicity to Mexican fruit fly (Diptera: Tephritidae). J Econ Entomol. 2001;94:150-6

42. Owusu HF, Jančáryová D, Malone D, Müller P. Comparability between insecticide resistance bioassays for mosquito vectors: time to review current methodology? Parasit Vectors. 2015;8:357. doi:10.1186/s13071-015-0971-6.

43. Lucht JM. Public acceptance of plant biotechnology and GM crops. Viruses. 2015;7:4254-81.

44. Nguyen TH, Nguyen HL, Nguyen TY, Vu SN, Tran ND, Le TN, et al. Field evaluation of the establishment potential of wMelPop Wolbachia in Australia and Vietnam for denque control. Parasit Vectors. 2015;8:563. doi:10.1186/s13071-015-1174-x.

45. Winskill P, Carvalho DO, Capurro ML, Alphey L, Donnelly CA, McKemey AR. Dispersal of engineered male Aedes aegypti mosquitoes. PLoS Negl Trop Dis. 2015:9:e0004156.

46. Hasspieler BM, Arnason JT, Downe AE. Toxicity, localization and elimination of the phototoxin, alpha-terthienyl, in mosquito larvae. J Am Mosq Control Assoc. 1988:4:479-84.

47. Nivsarkar M, Kumar GP, Laloraya M, Laloraya MM. Superoxide dismutase in the anal gills of the mosquito larvae of Aedes aegypti: its inhibition by alphaterthienyl. Arch Insect Biochem Physiol. 1991;16:249-55.

48. Volf $\mathrm{P}$, Volfova $\mathrm{V}$. Establishment and maintenance of sand fly colonies. J Vector Ecol. 2011;36 Suppl 1:S1-9.

49. Tsai CW, Rowhani A, Golino DA, Daane KM, Almeida RP. Mealybug transmission of Grapevine leafroll viruses: an analysis of virus-vector specificity. Phytopathology. 2010;100:830-4.

50. Akey DH, Beck SD. Continuous rearing of the pea aphid, Acyrthosiphon pisum, on a holidic diet. Ann Ent Soc Am. 1971;64:353-6.

51. Hung CH, Chang GF, Kumar A, Lin GF, Luo LY, Ching WM, et al. m-Benziporphodimethene: a new porphyrin analogue fluorescence zinc(II) sensor. Chem Commun (Camb). 2008:8:978-80

52. Drazen JM. Califf for the FDA. New Engl J Med. 2016;374:176-7.

53. Shelby A, Ernst K. Story and science: how providers and parents can utilize storytelling to combat anti-vaccine misinformation. Hum Vaccine Immunother. 2013:9:1795-801.

\section{Submit your next manuscript to BioMed Central and we will help you at every step:}

- We accept pre-submission inquiries

- Our selector tool helps you to find the most relevant journal

- We provide round the clock customer support

- Convenient online submission

- Thorough peer review

- Inclusion in PubMed and all major indexing services

- Maximum visibility for your research

Submit your manuscript at www.biomedcentral.com/submit
Biomed Central 